\title{
L'Equipement sensoriel des Trechinae souterrains (Coléoptères). II - Ultrastructure des trichobothries de l'Elytre
}

\author{
par \\ Christian JUBERTHIE et Françoise PIQUEMAL*
}

The sensoral outfit of subterranean Trechinae
II - Ultrastructure of the Elytral trichobothria

Summary

The ultrastructure of trichobothria $(\mathrm{Tm} 2, \mathrm{Tr} 2, \mathrm{Tr} 4)$ of the elytra has been studied in the troglobitic Coleoptera Geotrechus vulcanus and Aphaenops cerberus. Two bipolar neurons innervate these trichobothria. The first ends at the level of the hair base, and its distal segment contains a tubular body, characteristic of mechanoreceptive bristles. The other does not possess a tubular body, and its distal segment ends in the bristle canal; its function is unknown. The trichobothria possess one glial enveloping cell, one trichogen cell, and one tormogen cell; the latter two show an apical, common, large, receptor lymph cavity.

The small trichobothria are innervated by a large mechanoreceptor neuron, and by 4 smaller neurons; its function is unknown.

The trichobothria of blind Trechinae are highly specialized. A cuticular cup enshrines the hair base; hair and cup move together. The large amplitude swaying movements of the hair are controlled by a spongious tissue around the cup.

The trichobothria of Trechinae and Periplaneta have the same type of cuticular dome-shaped structure, and differ from trichobothria that arise from a cavity in the cuticle.

\section{INTRODUCTION}

Dans un premier travail (1975) nous avons étudié au microscope électronique à balayage l'équipement sensoriel de la face supérieure de l'élytre de plusieurs Coléoptères Trechinae souterrains. Chaque élytre porte 10 soies longues et souples, et non 8, appelées fouets par Jeannel en 1925, et que nous avons assimilées aux trichobothries. Trois de ces trichobothries sont extrêmement longues; c'est l'une (tm2) des 4 trichobothries du groupe huméral innervée par le

* Laboratoire souterrain du C. N. R. S., 09410 Moulis, France. 
nerf médian, et deux ( $\operatorname{tr} 2$ et tr4) des 4 trichobothries du groupe postérieur innervées par le nerf radial; leur longueur peut atteindre les $3 / 5$ de celle de l'élytre chez Aphaenops cerberus.

Dans ce travail nous étudions l'ultrastructure des deux types de trichobothries que nous avons distingués d'après leur taille.

\section{MATÉRIEL ET MÉTHODE}

Les animaux étudiés sont des Coléoptères troglobies, Geotrechus vulcanus provenant de la grotte du Portel (Ariège) et Aphaenops cerberus provenant de la grotte de Liqué (Ariège). Les élytres sont détachées et coupées en plusieurs morceaux pour faciliter la pénétration du fixateur. Elles sont fixées au froid 1 à 2 heures dans le glutaraldéhyde à $2,9 \%$ dans le tampon phosphate de Soerensen à $0,1 \mathrm{M}$, puis au tétroxyde d'osmium à $2 \%$ dans le même tampon. L'inclusion est faite à l'Epon 812 normal ou dans des Epons dont les proportions sont modifiées de façon à le rendre dur ou ultra-dur. Les coupes, réalisées avec un couteau en diamant monté sur l'ultramicrotome Reichert $\mathrm{OMU}_{2}$, sont récupérées sur des grilles recouvertes d'un support de Parlodion; elles sont contrastées à l'acétate d'uranyle et au citrate de plomb et observées au microscope Sopelem du Laboratoire souterrain sous $50 \mathrm{kv} .^{1}$

\section{RÉSULTATS}

I - TRICHOBOTHRIES DE GRANDE TAILLE (Tm2, Tr2, Tr4).

\section{A. Geotrechus vulcanus.}

1. Structures cuticulaires (Fig. 2).

En microscopie photonique la trichobothrie apparaît cylindrique et ne s'élargit pas dans sa partie basale contrairement à celle de Periplaneta americana (Gnatzy, 1973).

La base de la soie est insérée dans une cupule articulaire par une ouverture d'un diamètre à peine supérieur au sien; cette cupule est percée d'une ouverture basale qui offre le passage aux deux dendrites sensoriels.

Soie et cupule cuticulaire sont implantées au centre d'une élevure circulaire du tégument dorsal, correspondant à la formation décrite sous le nom d'aréole par certains auteurs (Vachon, 1973). Un espace optiquement clair, recouvert d'une membrane souple, formant un dôme, sépare la cupule de l'aréole.

L'observation sous la loupe binoculaire de trichobothries soumises à de faibles déplacements d'air montre que c'est l'ensemble soie + cupule cuticulaire qui est mobile; la cupule cuticulaire joue le rôle d'une rotule; le poil bouge également dans la cupule cuticulaire mais ceci est peu perceptible.

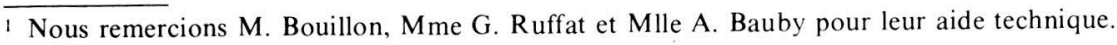


En microscopie électronique à balayage (Juberthie, Massoud et Piquemal, 1975), la couronne correspondant à l'espace optiquement vide est fermée à sa

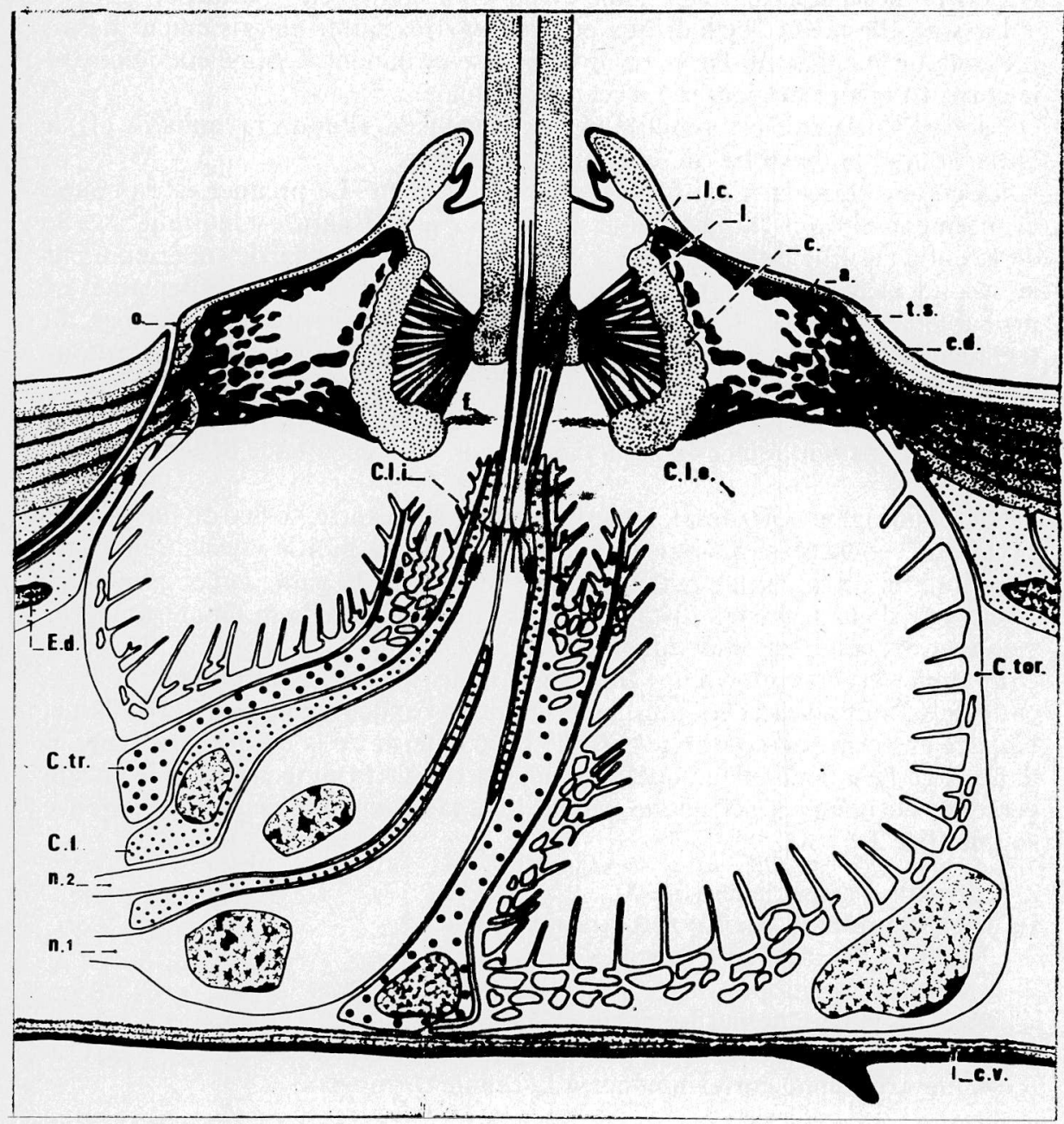

Fig. 1. Schéma d'une trichobothrie de grande taille de l'élytre d'Aphaenops cerberus et Geotrechus vulcanus. $a$. aréole, $c$. cupule cuticulaire, $c$. $d$. cuticule dorsale, C.e. première cellule enveloppe, $c . v$. cuticule ventrale, $c . l$. e. cavité lymphatique externe, $c . l$. $i$. cavité lymphatique interne, $C$. tor. cellule tormogène, $C$. tri. cellule trichogène, $c$. v. cuticule ventrale, $e$. $d$. épiderme de la face dorsale de l'élytre, $f$. fibres circulaires enchevêtrées formant un diaphragme ouvert, $l$. ligaments fibreux d'attache de la soie à la cupule, $l$. $c$. lame cuticulaire, $n .1$ neurone mécanorécepteur, $n .2$ second neurone; $o$. orifice d'une glande tégumentaire, $t$. $s$. tissu spongieux. 
partie supérieure par une membrane souple qui s'affaisse lors de la métallisation sous vide d'animaux non fixés au préalable.

En microscopie électronique à transmission, les observations montrent que l'insertion de la soie représente une disposition hautement spécialisée.

La soie elle-même, cylindrique et avec un très faible élargissement basal, présente un méplat sub-basal au niveau où se terminent la gaine cuticulaire et le corps tubulaire du neurone mécanorécepteur.

La base de la soie est attachée par des ligaments fibreux rayonnants (1.) à la paroi interne, bosselée, de la cupule cuticulaire.

La cupule cuticulaire ést formée de trois éléments. Le premier est la coupe proprement dite (c) formée d'une paroi épaisse, de nature identique à celle de la cuticule. Elle est suspendue à cette dernière par sa partie supérieure par le second élément qui est une mince lame cuticulaire (1.c.); cette lame est probablement souple. Le troisième élément est représenté par la partie du tégument auquel elle s'attache et qui forme une élevure en couronne autour de la soie. Cette élevure mesure le quart de l'épaisseur de la cuticule et correspond à peu près à l'exocuticule, son bord interne se replie à l'intérieur de la coupe, devient une mince lame de l'épaisseur de l'épicuticule et s'attache sur la soie.

Un diaphragme horizontal, lâche, ouvert en son centre, formé de fines fibres circulaires, enchevêtrées, (fig. 1/f.) apparaît dans la moitié inférieure de l'espace interne de la cupule cuticulaire, au niveau où la gaine cuticulaire commune aux deux segments distaux des neurones sensoriels se sépare pour envelopper chacun d'eux individuellement.

Le dôme (d) est formé d'une très mince lame, souple, correspondant à l'épicuticule et aux niveaux les plus superficiels de l'exocuticule. Sous cette lame, l'espace en forme de couronne entre le bord externe de la coupe cuticulaire et le bord de l'endocuticule auquel s'attache la cellule tormogène est vide en son centre et occupé à sa périphérie par un tissu lâche et spongieux en apparence souple (fig. $2 \mathrm{~b}$, t.s.).

2. Structures sensorielles (fig. 3).

La trichobothrie de Geotrechus vulcanus possède:

- 2 cellules sensorielles (neurones bipolaires),

- 3 cellules enveloppes,

- une première de nature gliale,

- une deuxième, correspondant à la cellule trichogène

- une troisième, correspondant à la cellule tormogène.

Fig. 2. Grande trichobothrie d'Aphaenops cerberus. (A) Coupe transversale des structures cuticulaires. $a$. aréole ou élevure de la face dorsale de l'élytre, $c$. cupule cuticulaire, $f$. partie périphérique des fibres qui attachent la base de la soie (s) au bord interne de la cupule cuticulaire, $c$. $l$. partie supérieure de la cavité lymphatique externe, $t$. $s$. éléments du tissu spongieux, mieux développé plus profondément. x 12.500. (B) Coupe longitudinale d'une partie de la zone d'insertion d'une trichobothrie, montrant la forme de la cupule cuticulaire (c.), la lame chitineuse (l.) qui attache cette dernière à la cuticule, les fibres (f.) qui rayonnent de sa face interne, la minceur de la cuticule au niveau du dôme $(d$.) comparée à l'épaisseur normale de la cuticule (c.u.), et le tissu spongieux (t. s.). x 11.500 . 
TRICHOBOTHRIES DE L'ELYTRE D'APHAENOPS ET GEOTRECHUS
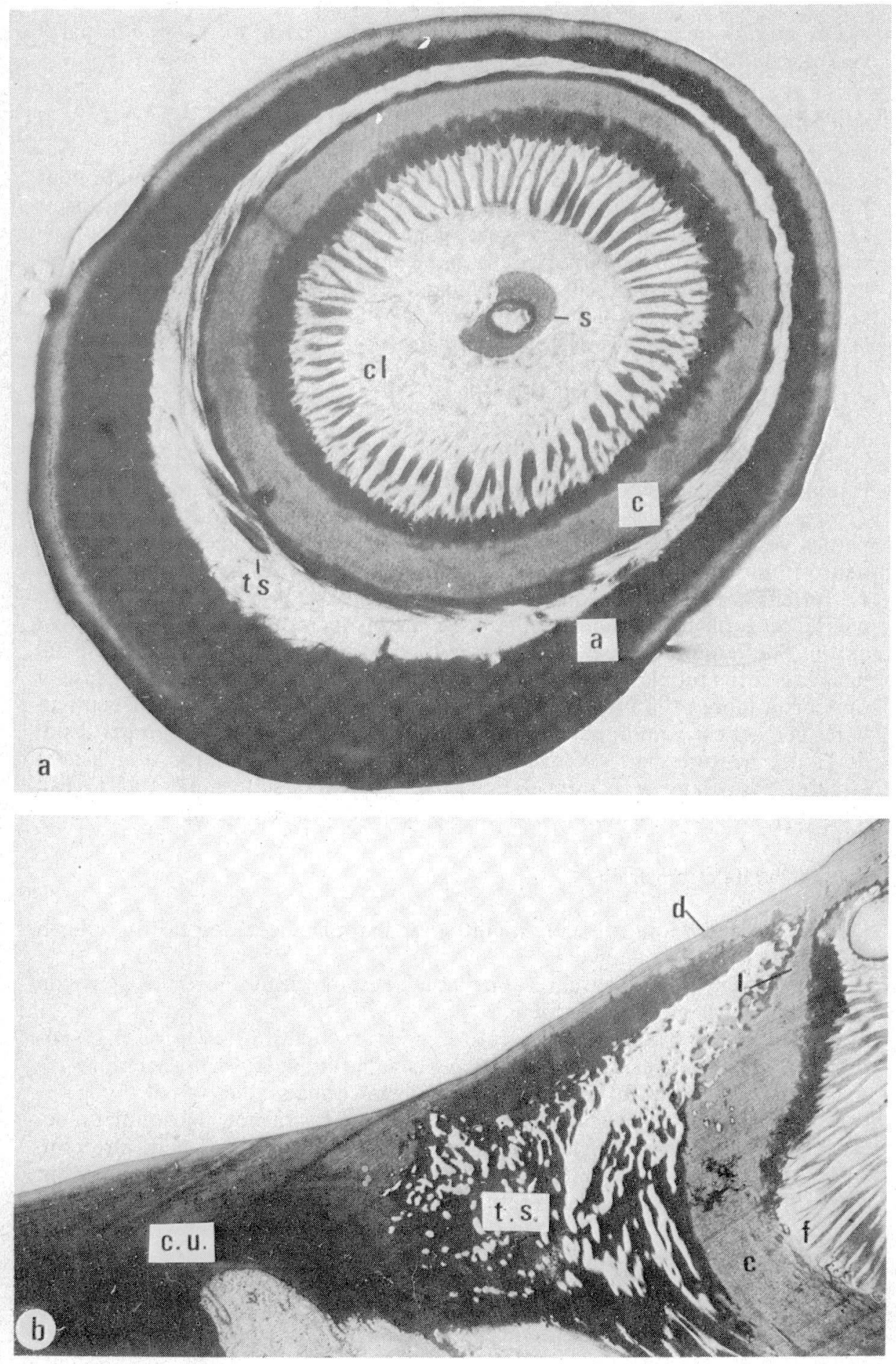
Ces cellules occupent tout l'espace qui sépare la paroi dorsale de la paroi ventrale de l'élytre.

a) Cellules sensorielles.

Chaque trichobothrie est innervée par deux neurones bipolaires, qui présentent l'organisation longitudinale classique en segments proximal, ciliaire et distal. L'un des neurones se termine assymétriquement dans la base de la soie et possède un corps tubulaire entouré de la gaine cuticulaire, caractéristique des cellules à fonction mécanoréceptrice (Thurm, 1965). L'autre neurone émet un segment distal qui se prolonge sans se ramifier dans la base due canal axial de la soie et ne présente pas de corps tubulaire.

Première cellule sensorielle (neurone mécanorécepteur).

Le cytoplasme est clair, riche en mitochondries ovoïdes à crêtes transversales; il renferme quelques éléments courts de réticulum endoplasmique granulaire, quelques polysomes, quelques dictyosomes périnucléaires qui élaborent des petites vésicules de $250 \mathrm{~A}^{\circ}$ de diamètre environ, quelques neurotubules. Les neurotubules deviennent nombreux dans le segment proximal où ils s'alignent. La formation ciliaire est composée de 2 centrioles de type $9+0$, qui donnent naissance à plusieurs racines ciliaires; chacun des doublets centriolaires est entouré de matériel dense. Les doublets se prolongent dans le segment distal jusqu'au corps tubulaire où ils sont noyés dans une masse de matériel dense et où leur nombre se multiplie. Le segment distal est entouré d'une gaine cuticulaire; celle-ci est commune dans sa partie basale avec celle du segment distal de l'autre neurone puis elle s'individualise rapidement. Le corps tubulaire et la gaine cuticulaire, pénètrent dans la base du poil et se terminent au niveau d'un méplat, ce qui met en contact la gaine avec la face externe de la soie.

Seconde cellule sensorielle.

Elle est identique à la première quant à son cytoplasme et son noyau; elle en diffère sur les points suivants:

- le segment distal est grêle et son diamètre est moitié moindre: $0,4 \mu$ environ contre 0,8 à $1 \mu$ pour la première;

- le segment distal est dépourvu de corps tubulaire; il se prolonge sans ramification dans le canal axial de la soie; la gaine cuticulaire qui l'accompagne se fond avec la paroi du canal et cesse' d'être reconnaissable;

- les deux centrioles sont de type $9+0$. Le plus souvent, un doublet n'occupe pas une position parfaitement axiale ni périphérique; il peut être considéré comme une paire de tubules périphériques déplacée traduisant un début de perte de la symétrie rayonnée.

b) Première cellule enveloppe. 
Les deux cellules sensorielles sont complètement enveloppées, depuis leur corps cellulaire jusqu'à la partie basale des segments distaux et de la gaine cuticulaire, par une cellule enveloppe (c.1.).

Le noyau et le cytoplasme périnucléaire de la cellule enveloppe sont en partie enchâssés entre les deux corps cellulaires sensoriels à peu près au niveau du noyau du neurone mécanorécepteur. La cellule enveloppe envoie trois lames cytoplasmiques; la première entre les deux neurones, et les deux autres latéralement; elles entourent chaque cellule sur un tour ou un tour et demi. De 0,25 à $0,5 \mu$ d'épaisseur autour des péricaryones sensoriels, les lames latérales forment une couronne cytoplasmique de $1 \mu$ d'épaisseur près de la base des segments distaux, et de 3 à $4 \mu$ de diamètre.

Le noyau mesure de 5 à $8 \mu$ et renferme une chromatine clairsemée, poudreuse, avec quelques mottes accolées à l'enveloppe nucléaire.

Le cytoplasme périnucléaire renferme d'assez nombreux éléments du réticulum endoplasmique granulaire, de nombreux ribosomes libres, des polysomes, et de rares dictyosomes peu actifs. Les mitochondries sont peu nombreuses, petites, à crêtes transversales; les corps myéliniformes ne sont pas rares, des microtubules épars parcourent le cytoplasme.

Près des zones centriolaires, le nombre des microtubules augmente considérablement, et de 5 à 6 hémidesmosomes assurent l'attache du segment proximal de chacun des deux neurones avec les lames de la cellule enveloppe.

$\mathrm{Au}$ niveau de chaque zone ciliaire, la cellule enveloppe laisse un petit espace correspondant à l'“innerer liquorum rezeptor lymphraum" des auteurs allemands et à l'"inner receptor lymph cavity" des auteurs anglo-saxons. ${ }^{1}$

A sa partie distale un espace de 500 à $800 A^{\circ}$ sépare sa membrane plasmique de celle des deux neurones; cet espace est en partie occupé par la base de la gaine cuticulaire qui mesure $300 \mathrm{~A}^{\circ}$ d'épaisseur en moyenne, et atteint localement $500 \mathrm{~A}^{\circ}$.

Chez un adulte, peu de temps après la mue imaginale et n'ayant pas encore atteint sa pigmentation définitive, nous avons observé sur la membrane plasmique qui borde la gaine cuticulaire de nombreuses figures en "omega"; certaines, par leur aspect hérissé, correspondent à des vésicules de pinocytose, d'autres, absolument lisses, renferment une ou plusieurs inclusions denses aux électrons et semblent représenter la libération d'un matériel qui participerait à l'élaboration de la gaine cuticulaire.

c) Cellule trichogène.

\footnotetext{
1 Certains auteurs ont conservé en français le terme de vacuole, pour l'espace ménagé entre la partie apicale des cellules accessoires du récepteur et la base de la soie. Ce terme est inadéquat et ne permet pas de rendre compte de l'existence de deux cavités successives, la première la plus interne autour de la région centriolaire, la seconde à la base de la soie. En l'absence de terme précis, nous proposons donc d'harmoniser la terminologie française avec celles proposées par les auteurs allemands et anglais et d'utiliser les termes de "cavité lymphatique interne" pour la première, la plus interne, et "cavité lymphatique externe" pour la seconde.
} 


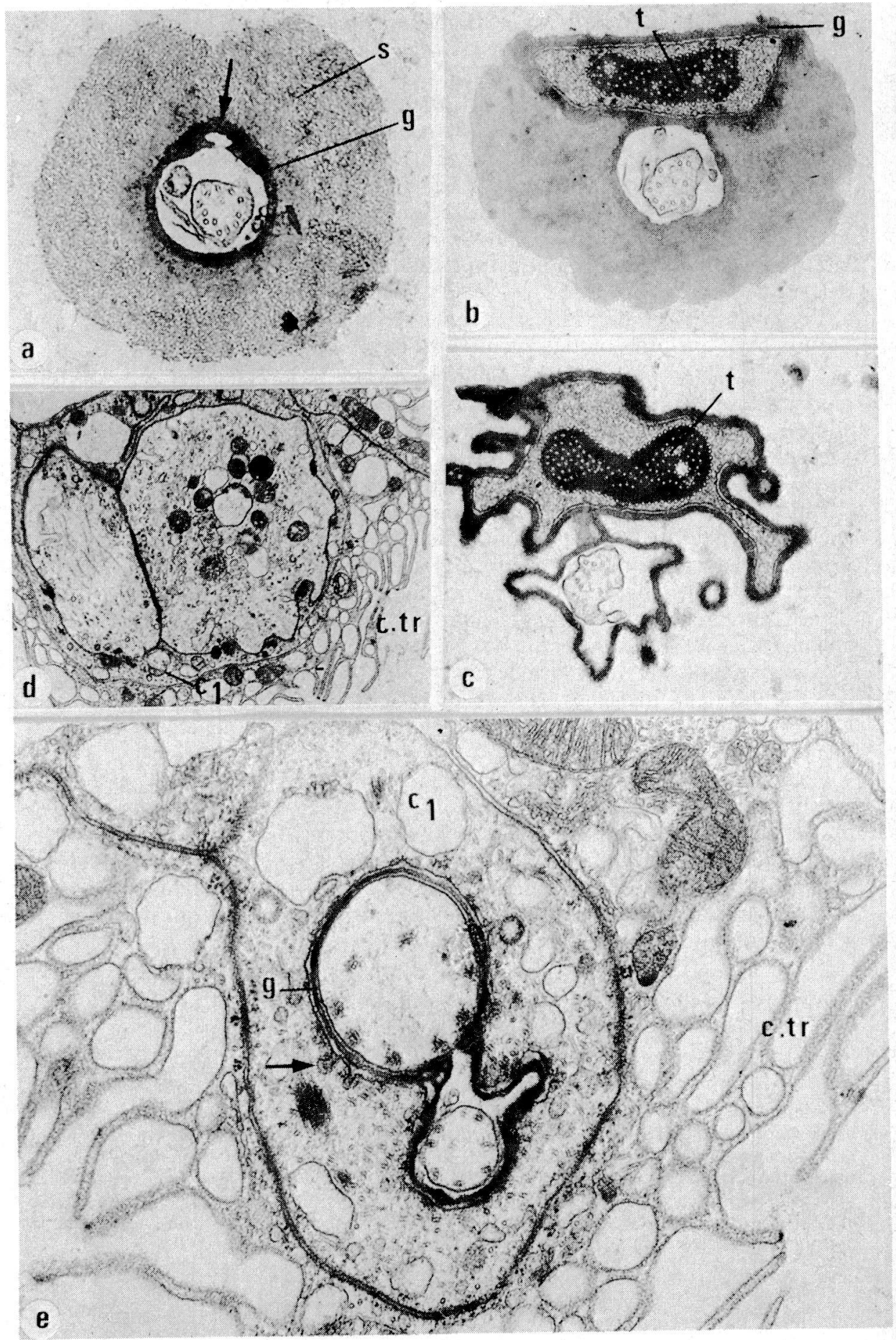


Fig. 3. Grande trichobothrie de Geotrechus vulcanus, coupes transversales. (A) Base de la soie (s.) et extrémité de la gaine cuticulaire (g.) renfermant un segment distal dépourvu de corps tubulaire et qui se digite; la flèche souligne la partie distale de la gaine qui correspond à l'autre neurone qui apparaît sur la coupe suivante plus basale. x 31.000. (B) Coupe encore plus basale où l'on reconnaît au centre le segment distal précédent avec ses digitations, et d'un côté l'autre segment distal entouré de sa gaine cuticulaire (g.) et renfermant un corps tubulaire caractéristique (t.) x 29.000. (C) La gaine qui enveloppe chacun des deux segments distaux s'individualise; un seul segment distal renferme un corps tubulaire $x$ 26.000. (D) Coupe des segments proximaux des deux neurones sensoriels; ils sont enveloppés par la première cellule enveloppe (c. 1), à la membrane interne de laquelle ils sont reliés par hémidesmosomes; $C$. $t r$., cellule trichogène x 11.000 . (E) Coupe au niveau de la région centriolaire des deux neurones; le gros dendrite appartient au neurone mécanorécepteur; la gaine (g.) encore présente se termine à ce niveau; une première cellule (C. 1) enveloppe les 2 neurones et sa membrane interne présente des figures en "omega" (Flèche); elle est entourée par la cellule trichogène (C. tr.) formée d'une mince couche cytoplasmique, d'un réseau en nid d'abeille et de lames cytoplasmiques qui se projettent dans la cavité lymphatique externe; cellule enveloppe et cellule trichogène sont reliées par une longue jonction septée $\mathrm{x} 41.000$.

La cellule enveloppe (C.1.) est entièrement entourée par une cellule correspondant à la cellule trichogène (C. tr.). Celle-ci est subcylindrique, son noyau est basal, sa partie apicale est creusée d'une cavité étroite en forme de couronne qui contribue à la formation de la cavité lymphatique externe. Le fond de cette cavité est tapissé d'une formation en nid d'abeille qui se transforme en cloisons courtes, de 500 à $600 \mathrm{~A}^{\circ}$ d'épaisseur, qui se projettent dans la cavité. Son cytoplasme renferme des mitochondries allongées à crêtes transverses, d'assez nombreux microtubules, des vésicules du réticulum endoplasmique lisse, et des polysomes épars.

\section{d) Cellule tormogène.}

La cellule tormogène (C. tor.) occupe toute la hauteur de l'espace interne de l'élytre; elle est contigue à l'hypoderme très mince de la face ventrale, et à celui nettement plus épais de la face dorsale, auquel elle est attachée par des desmosomes septés. Au niveau de l'attache de la cellule au tégument, la cuticule envoie quelques courtes expansions lamelliformes, de $400 \mathrm{~A}^{\circ}$ de large et de 0,2 à $0,4 \mu$ de long.

Elle a la forme d'une ampoule, traversée par l'ensemble constitué des deux neurones sensoriels, de la cellule enveloppe (C.1) et de la cellule trichogène (C. tr.).

Elle est creusée d'une vaste cavité qui s'ouvre à la base de la coupe cuticulaire de la soie et qui, fusionnée avec celle de la cellule trichogène, forme la cavité lymphatique externe du récepteur (outer receptor lymph cavity). Le cytoplasme est disposé en une mince couche périphérique; les replis de sa paroi interne forme un nid d'abeille qui se résoud en cloisons de 500 à $600 \mathrm{~A}^{\circ}$ d'épaisseur et de 1 à $3 \mu$ de longueur, lesquelles se projettent dans la cavité lymphatique externe.

Son cytoplasme renferme un nombre assez élevé de mitochondries allongées, à nombreuses crêtes transverses. Le réticulum endoplasmique granulaire est 
représenté par des éléments épars, assez courts, dont certains sont en relation avec le feuillet externe de l'enveloppe nucléaire. Les dictyosomes élaborent des nuages de petites vésicules. Des polysomes sont épars dans le cytoplasme ainsi que des microtubules.

Le noyau est ovoïde et mesure $3 \mu$ sur $6 \mu$; sa chromatine est peu abondante, poudreuse avec quelques amas accolés à la membrane nucléaire.

\section{B. Aphaenops cerberus.}

Nous avons étudié la trichobothrie de grande taille $\mathrm{Tr}_{2}$.

Sa structure est identique à celles des trichobothries de grande taille de Geotrechus vulcanus; en particulier, comme celles-ci, elle présente deux neurones sensoriels dont l'un renferme un corps tubulaire; l'autre en est dépourvu et se prolonge dans le canal de la soie.

\section{II - TRICHOBOTHRIES DE PETITE TAILLE.}

\section{A. Aphaenops cerberus (Fig. 4 et 5).}

Les trichobothries de petite taille sont représentées par les soies humérales $\mathrm{tm}_{1}$, $\mathrm{tm}_{3}, \mathrm{tm}_{4}$ innervées par le nerf médian, les soies postérieures $\operatorname{tr}_{1}$ et $\operatorname{tr}_{3}$, innervées par le nerf radial, la soie antérieure ta innervée par la branche anale du nerf cubito-anal, et la soie tout-à-fait postérieure tc innervée par la branche cubitale du nerf cubito-anal.

Nous avons étudié les trichobothries $\mathrm{tm}_{1}$ et $\mathrm{tm}_{4}$.

Hormis la taille, le schéma d'organisation de ces petites trichobothries est identique à celui des grandes. On retrouve, en particulier, le mode d'insertion de la soie, la coupe cuticulaire et les différenciations cytologiques qui lui sont associées, la première cellule enveloppe (C. 1) entourée d'une cellule trichogène (C. tr.), elle-même enveloppée d'une cellule tormogène (C. tor.); ces cellules ont le même aspect cytologique que dans le cas précédent.

Fig. 4. Petite trichobothrie d'Aphaenops cerberus, coupes transversales. (A) Base de la soie (s.) et extrémité de la gaine cuticulaire $(g$. $)$ x 39.000. (B et C) Coupe du corps tubulaire $(t$.) à deux niveaux, le premier tout-à-fait à la base de la soie; le segment distal renfermant le corps tubulaire et les 4 autres segments distaux dépourvus de corps tubulaires sont bien visibles en $\mathrm{C}$ : une gaine cuticulaire commune (g.) entoure les 5 dendrites. $x$ 43.500. (D) Coupe plus basse que la précédente; la gaine cuticulaire enveloppe, d'une part le gros neurone mécanorécepteur, et d'autre part les 4 autres neurones. $x$ 43.500. (E) Coupe au niveau de l'extrémité de la première cellule enveloppe (C. 1$)$ et de la cellule trichogène $(C$. $t r$. $)$; la gaine (g.) est encore présente. $x$ 43.500. (F) Coupe au niveau de la région centriolaire des 4 petits neurones, montrant la cavité lymphatique interne (i.); le gros neurone est attaché à la membrane interne de la première cellule enveloppe par des desmosomes $(d) ;$.$C . t r$. et C. to. cellule trichogène et tormogène avec leurs lamelles se projetant dans la cavité lymphatique externe. $x$ 18.000. (G) Coupe au niveau des segments proximaux des 5 neurones (1 à5); le neurone mécanorécepteur (1) est toujours relié à la première cellule enveloppe (C. 1) par des desmosomes; cette cellule envoie des microviilosités dans la cavité lymphatique interne. $x 14.500$. 

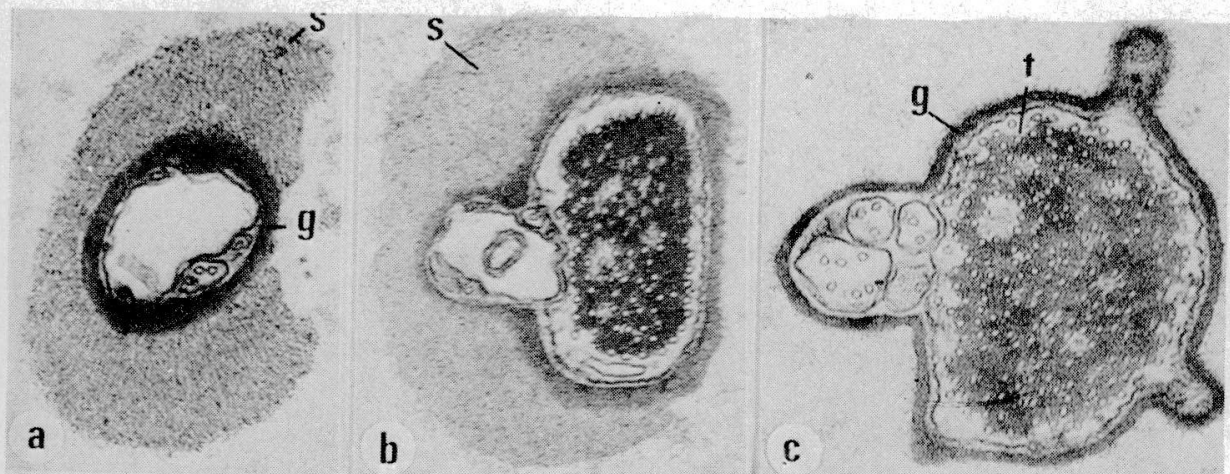

a

b
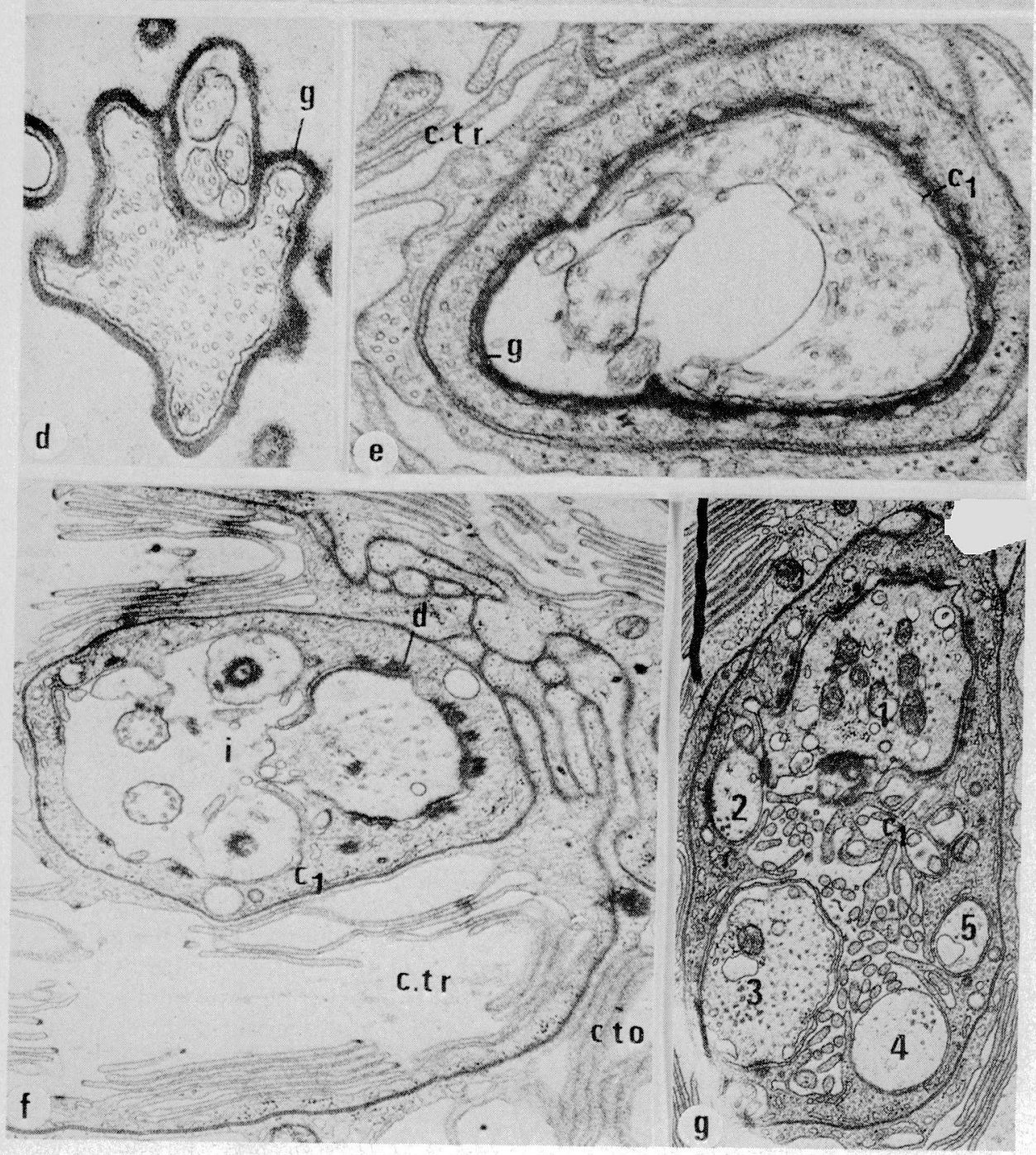


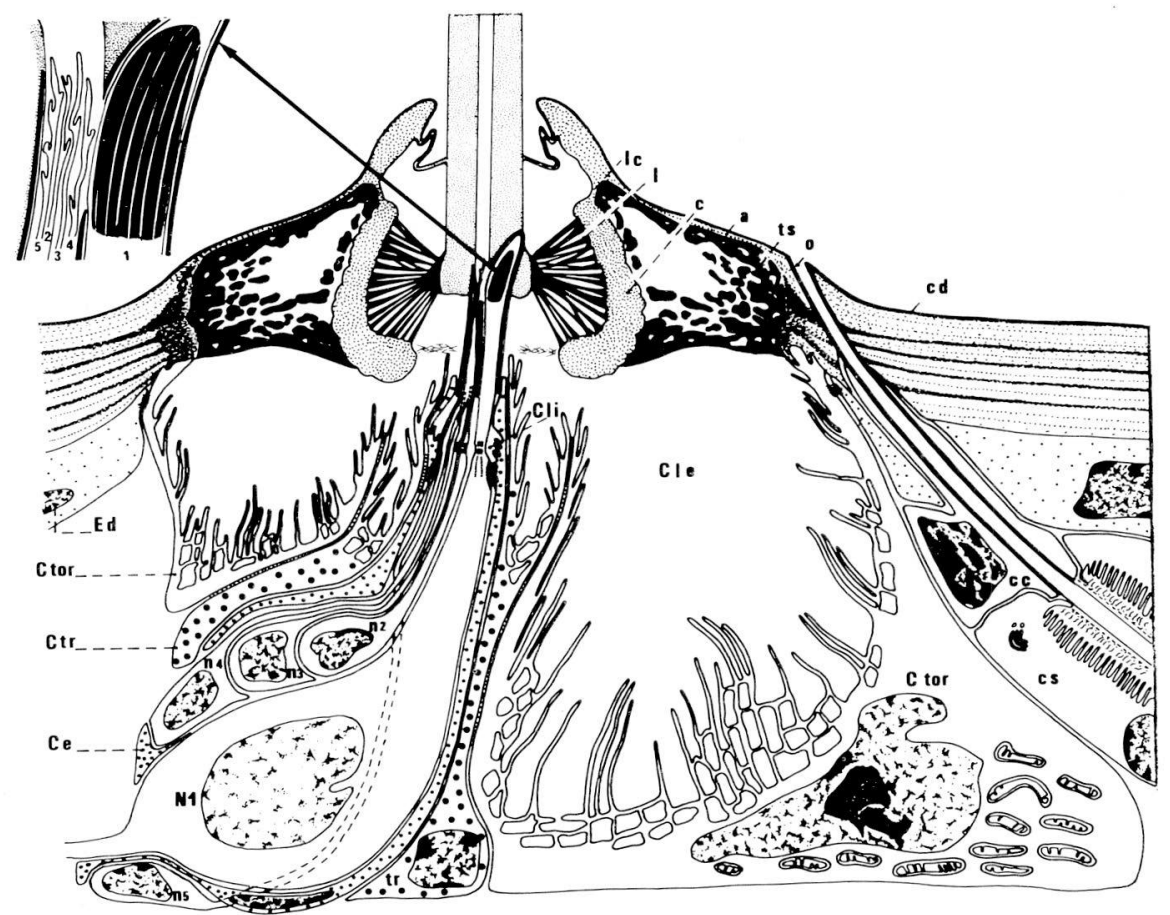

Fig. 5. Schéma d'une trichobothrie de petite taille (tm4) d'Aphaenops cerberus. Même légende que la figure 1 et: $\mathrm{n} 2, \mathrm{n} 3, \mathrm{n} 4, \mathrm{n} 5$, petits neurones sensoriels; $C$. $C$. = cellule du canal de l'une des glandes tégumentaires qui s'ouvre à la base de la trichobothrie; $C$. $S$. = cellule sécrétrice de cette glande. Schéma de détail, en haut à gauche, des terminaisons des segments distaux des neurones sensoriels mécanorécepteur (1) et autres (2 à 5).

La différence essentielle réside dans l'innervation de la trichobothrie. Au neurone mécanorécepteur s'adjoint un nombre plus élevé de neurones dépourvus de corps tubulaires. Ils sont au nombre de 4 dans la soie $\mathrm{tm}_{4}$. La première cellule enveloppe (C. 1) ménage au niveau des zones centriolaires une cavité lymphatique interne assez importante, et projette des microvillosités dans la partie basale de cette dernière.

Le neurone mécanorécepteur est beaucoup plus volumineux que les 4 autres neurones et il renferme un corps tubulaire bien développé. Les péricaryones des 4 petits neurones sont accolés au gros neurone; chacun est enveloppé par une lame cytoplasmique provenant de la cellule enveloppe C.1. Chacun de ces neurones émet un segment proximal, et, après la zone ciliaire, un sẹul segment distal; ce dernier bourgeonne de très courtes digitations dans sa partie terminale, et se termine dans la base du canal de la trichobothrie. Le violet crystal ne pénètre pas dans la trichobothrie, ce qui semble indiquer l'absence de pore terminal. 


\section{CONCLUSIONS}

1. Les trichobothries grandes et petites de Geotrechus vulcanus et d'Aphaenops cerberus bougent au moindre courant d'air; c'est d'une part l'ensemble poil et cupule qui enchâsse la base du poil qui oscille, la cupule faisant office de rotule, et d'autre part, à un degré moindre le poil lui même dans la cupule.

2. L'aspect externe de l'embase des trichobothries est identique chez tous les Trechinae observés et il est à quelques détails près, semblable à celle des "fadenhaare" ou "thread setae" des cerques de Periplaneta americana (Nicklaus, Lundquist et Wersall, 1968, Gnatzy, 1973, Counter, 1976).

3. La partie cuticulaire des trichobothries des Trechinae souterrains est nettement plus élaborée que celle de Periplaneta, telle qu'elle est décrite par Gnatzy (1973). En premier, on retrouve des formations communes: l'aréole correspond à la "cuticulare Kuppel", la cupule cuticulaire au "cuticularer zylinder"; aréole et "cuticulare Kuppel" ont une paroi épaisse dans la zone qui enserre le poil, et une paroi mince dans la zone périphérique. On note, ensuite, des différences importantes: le cylindre cuticulaire de Periplaneta est beaucoup plus développé chez Aphaenops et Geotrechus, il se referme presque ventralement et prend la forme d'une coupe d'où l'utilisation du terme de cupule cuticulaire; la base du poil des Trechinae ne s'élargit pas notablement et ne donne pas naissance à une plaque basale "Haarbasisplatte", qui chez Periplaneta se meut dans le cylindre cuticulaire lorsque le poil oscille; en revanche un réseau important de ligaments fibreux, probablement élastiques, rayonnants, s'est fortement développé chez les Aphaenops et attache la base du poil à la face interne de la cupule cuticulaire; la lame cuticulaire qui recouvre la partie en dôme optiquement vide est beaucoup plus mince chez les Trechinae que chez Periplaneta et donc plus souple, elle mesure de $1 / 20$ à $1 / 15$ de l'épaisseur de la cuticule contre $1 / 4$ environ chez Periplaneta; une couronne de tissu, lâche, spongieux et fibreux, enrobe chez les Trechinae la face externe de la cupule sur une hauteur sensiblement égale à celle de l'endocuticule et de l'exocuticule réunies, auxquelles ce tissu s'attache également; du fait de la présence de ce tissu spongieux l'attache sur la cuticule de la paroi de la cellule tormogène $\left(\mathrm{H}_{3}\right.$ de Gnatzy, fig. 2a) est rejetée à la périphérie au niveau où le tégument retrouve son épaisseur normale.

On peut en conclure que l'allongement considérable des trichobothries qui caractérise les Trechinae troglobies, tels que les Aphaenops. et les Geotrechus par rapport aux espèces endogées ou épigées ( 3 d'entre elles atteignant les $3 / 5$ de la longueur des élytres chez $A$. cerberus) s'accompagne de modifications et de néoformations cytologiques dont l'une des plus caractéristiques est le développement d'un tissu spongieux dont le rôle serait d'amortir les oscillations de la cupule cuticulaire, devenue extrêmement mobile dans ce type de trichobothrie.

4. Les trichobothries des élytres des Trechinae et des cerques de Periplaneta 
americana appartiennent au même type de structure cuticulaire, celles des Trechinae étant plus différenciées. Ce type, à dôme cuticulaire membraneux, se différencie des trichobothries classiques qui sont implantées dans des coupes tégumentaires largement ouvertes à une ou plusieurs chambres, et qui ont été décrites chez de nombreux Insectes, notamment chez les Orthoptéroides Grillus bimaculatus (Gnatzy et Schmidt, 1971, Schmidt et Gnatzy, 1971) et Acheta domesticus (Edwards et Palska, 1974; Counter, 1976), chez les Hẻmiptéroides (Carayon et Villiers, 1968, Carayon, 1970), Pyrrhocoris apterus et Dysdercus intermedius (Gaffal, 1976), chez les Arachnides, Scorpions (Vachon, 1973), Araignées (Christian, 1971), Acariens (Haupt et Coineau, 1975), les Myriapodes Symphyles (Haupt, 1970) et Pauropodes (Haupt, 1976).

5. Les grandes trichobothries d'A. cerberus et de $G$. vulcanus sont innervées par 2 neurones sensoriels bipolaires, les petites trichobothries par 5 neurones.

6. Les trichobothries des Trechinae assurent une fonction mécanoréceptrice par l'un de leur neurone car il renferme un corps tubulaire dans son segment distal et se termine dissymétriquement dans la base de la soie. Ce neurone est toujours le plus volumineux et la fonction mécanoréceptrice semble prépondérante. La fonction du second neurone des trois grandes trichobothries et des 4 autres neurones des sept petites trichobothries reste indéterminée; l'absence de corps tubulaire et le fait que les segments distaux pénètrent au moins dans la partie proximale du canal de la soie plaiderait pour une fonction chémoréceptrice ou hygroréceptrice. Cependant, en microscopie électronique nous n'avons pas pu déceler la présence de pores dans la paroi de la soie ni à son extrémité; de même, l'utilisation du violet crystal ne nous a pas conduit à mettre en évidence la pénétration du colorant dans la soie.

Les trichobothries des Trechinae diffèrent donc des "Fadenhaare" ou "threadhairs" des cerques des Orthoptères par le fait que ces derniers ne possèdent qu'un seul neurone qui est de type mécanorécepteur. Des trichobothries d'autres groupes sont, cependant, innervées par plusieurs neurones; il en est ainsi pour les trichobothries des tarses et des métatarses de l'Araignée Tegenaria derhami qui ont 4 neurones dont un seul forme un corps tubulaire typique (Christian, 1971), chez les Myriapodes Symphyles Scutigerella immaculata (Haupt, 1970) dont les deux trichobothries possèdent 16 neurones et 32 terminaisons dendritiques chacune pourvue d'un corps tubulaire, et chez les Pauropodes Allopauropus sp. (Haupt, 1976) où elles en possèdent 8 .

Par ailleurs, l'innervation de mécanorécepteurs par 2 neurones bipolaires, dont 1 seul renferme un corps tubulaire et dont l'autre se termine dans la base du canal de la soie, a été décrite chez des Sensilla chaetica de la tête de Locusta migratoria (Gaffal, Tichy, Theiss, Seelinger, 1975).

7. La première cellule enveloppe (c. 1) a les propriétés d'une cellule gliale par les lames cytoplasmiques qu'elle envoie autour des neurones bipolaires, mais, par ailleurs, elle secrète la gaine cuticulaire. Elle est l'homologue de la première cellule enveloppe (Hüllzelle 1, H 1) des "fadenhaare" des cerques de Grillus 
bimaculatus et de Periplaneta americana. Chez cette dernière espèce Gnatzy (1976) appelle cette cellule "dendritic sheath cell", et les "thread hairs" possèdent, de plus, deux cellules gliales plus internes qui enveloppent le corps du neurone bipolaire mais ne participent pas à la formation de la gaine cuticulaire. Elle est l'homologue de la "neurilemme cell" décrite par Blaney et Chapman (1969), et par son double rôle, de la cellule de Schwann qui entoure la cellule nerveuse bipolaire et sécrète la gaine cuticulaire des stylets maxillaires de Rhodnius prolixus.

Les deux cellules qui forment à leur extrémité la cavité lymphatique externe (outer receptor lymph cavity) sont l'homologue des cellules enveloppes moyenne (H2) et externe (H3) des "fadenhaare" précédents, et correspondent respectivement à la cellule trichogène et à la cellule tormogène. Un trait caractéristique d'Aphaenops et de Geotrechus est le volume considérable de la cavité lymphatique externe du récepteur, la cellule tormogène acquérant une forme de cupule à paroi mince.

8. Une petite protubérance cutıculaire sur la face interne de la cupule et sur laquelle s'attachent des fibres circulaires est présente; elle semble similaire à la formation décrite par Gnatzy (1976) dans le cylindre chitineux, ou "hair base", des "thread hairs" des cerques de Periplaneta americana. D'après cet auteur la présence de cette formation influerait en orientant l'inclinaison de la soie, et en intervenant par la déformation du corps tubulaire qu'elle peut provoquer lorsque la soie s'incline dans la production d'un signal, et permettrait de reconnaître la face de dépolarisation. Sa position chez Aphaenops cerberus en face du corps tubulaire plaide pour un rôle similaire, les fibres circulaires enchevêtrées qui en partent pouvant également intervenir.

\section{RÉSUME}

Les trichobothries de grande taille $(\operatorname{Tm} 2, \operatorname{Tr} 2, \operatorname{Tr} 4)$ de Geotrechus vulcanus et Aphaenops cerberus sont innervées par deux neurones bipolaires. L'un se termine assymétriquement à la base du poil et présente un corps tubulaire dans son segment distal, ce qui lui confère une fonction mécanoréceptrice. L'autre ne présente pas de corps tubulaire et son segment distal se prolonge sans se rámifier au moins dans la partie proximale du canal du poil; sa fonction reste inconnue. Les deux neurones sont enveloppés par une cellule enveloppe, de nature gliale et qui sécrète la gaine cuticulaire. Cette cellule est entourée par une cellule trichogène, elle-même enveloppée par une cellule tormogène; cellules trichogène et tormogène sont creusées d'une grosse cavité, tapissée de lamelles, qui entoure la base du poil et qui correspond à la cavité lymphatique externe.

Les trichobothries de petite taille sont innervées par un gros neurone mécanorécepteur et par 4 neurones plus petits dont la fonction n'est pas connue.

Les trichobothries des Trechinae souterrains sont des organes hautement spécialisés qui présentent des dispositions cuticulaires originales. Une cupule cuticulaire enchasse la base du poil et l'ensemble poil + cupule est mobile, la cupule jouant le rôle d'une rotule; le poil bouge également dans la cupule. Les balancements de grandes amplitudes sont amortis par un tissu spongieux qui entoure la cupule.

Les trichobothries des Trechinae et les "fadenhaare" des cerques de Periplaneta ont en commun le même type de structure cuticulaire, en dôme membraneux, ce qui les différencie des trichobothries classiques implantées dans une coupe de la cuticule. 


\section{BIBLIOGRAPHIE}

BARTH, F. G.: Sensory information from strains in the exoskeleton. The Insect Integument. Ed. H. R. Hepburn 445-473 (1976).

BLANEY, W. M., CHAPMAN, R. F.: The fine structure of the terminal sensilla on the maxillary palps of Schistocerca gregaria (Forskal) (Orthoptera, Acrididae). Z. Zellforsch. 99, 74-97 (1969).

CARAYON, J., VILliERS, A.: Etude sur les Hémiptères Pachynomidae. Ann. Soc. Ent. Fr. (N. S.) 4, 3, 703-739 (1968).

CARAYON, J.: Etude des Alloeorhynchus d'Afrique centrale avec quelques remarques sur la classification des Nabidae (Hemiptera). Ann. Soc. Ent. Fr. (N. S.) 6, 4, 899-931 (1970).

CHRISTIAN, U.: The ultrastructure of the trichobothria of the house spider Tegenaria derhami (Scopoli), (Agelenidae, Araneae). Cytobiologie 4, 2, 172-185 (1971).

COUNTER, S. A.: An electrophysiological study of sound sensitive neurons in the "Primitive ear" of Acheta domesticus. J. Insect Physiol. 22, 1-8 (1976).

EDWARDS, J. S., PALKA, J.: The cerci and abdominal giant fibers of the house cricket, Acheta domesticus - I. Anatomy and physiology of normal adults. Proc. R. Soc. London(B) 185, 83-103 (1974).

GAFFAL, K. P.: Die Feinstruktur der Sinnes- und Hüllzellen in den antennalen Schmecksensillen von Dysdercus intermedius Dist. (Phyrrhocoridae, Heteroptera). Protoplasma 88, 101115 (1976).

GAFFAL, K. P., TICHY, H., THEISS, J. et SEELINGER, G.: Structural polarities in Mechanosensitive Sensilla and their influence on Stimulus transmission (Arthropoda). Zoomorph. 82, 79-103 (1975).

GNATZY, W., SCHMIDT, K.: Die Feinstruktur der Sinneshaare auf den cerci von Grillus bimaculatus Deg. (Saltatoria, Gryllidae). 1. Die Faden und Keulenhaare. Z. Zellforsch. 122, 190-209 (1971).

GNATZY, W.: Die Feinstruktur der Fadenhaare auf den cerci von Periplaneta americana L. Verhand. Deutsch. Zool. Gesellsch. 66, 37-41 (1973).

GNATZY, W.: The ultrastructure of the Thread-hairs on the cerci of the Cockroach Periplaneta americana $\mathrm{L}$. The intermoult phase. J. Ultrastruct. Res. 54, 124-134 (1976).

HAUPT, J.: Beitrag zur Kenntnis der Sinnesorgane von Symphylen (Myriapoda) 1. Elektronenmikroskopische Untersuchung des Trichobothriums von Scutigerella immaculata Newport. Z. Zellforsch. 110, 588-599 (1970).

HAUPT, J.: Anpassung an einen Lebensraum: das hygrophile Edaphon. Gesellsch. Natur. Freunde, Berlin 16, 2, 89-97 (1976).

HAUPT, J., COINEAU, Y.: Trichobothrien und Tastborsten der Milbe Microcaeculus (Acari, Prostigmata, Caeculidae). Z. Morph. Tiere 81, 305-322 (1975).

JEANnel, R.: Morphologie de l'élytre des Coléoptères adephaga. Arch. Zool. exper. gén., 64, 1-84 (1925).

JUBERTHIE, C., PIQUEMAL, F., MASSOUD, Z.: Equipement sensoriel des Coléoptères souterrains Trechinae. I. Etude au microscope à balayage de l'élytre d'Aphaenops et de Geotrechus. Ann. Spéléol. 30, 3, 539-551 (1975).

NICKLAUS, R., LUNDQUIST, P. G., WERSÄLL, J.: Die Übertragung des Reizes auf den distalen Forsatz der Sinneszelle bei den Fadenhaaren von Periplaneta americana. Verh. Deutsch. Zool. Ges. 61, 578-584 (1968).

SCHMIDT, K., GNATZY, W.: Die Feinstruktur der Sinneshaare auf den cerci von Grillus bimaculatus Deg (Saltatoria, Gryllidae). II Die Hautung der Faden und Keulenhaare. $Z$. Zellforsch. 122, 210-226 (1971).

VACHON, M.: Etude des caractères utilisés pour classer les familles et les genres de Scorpions (Arachnides). 1. La trichobothriotaxie en Arachnologie. Sigles trichobothriaux et types de trichobothriotaxie chez les Scorpions. Bull. Mus. Nat. Hist. nat. Paris, 3e ser. 140, 857-958 (1973). 